\title{
FEM-BASED AUTOMATIC SEGMENTATION OF MUSCLE AND FAT TISSUES FROM THORACIC CT IMAGES
}

\author{
Karteek Popuri $^{a}$, Vickie Baracos ${ }^{b}$, Nina Esfandiari $^{b}$, Martin Jägersand $^{a}$, Dana Cobzas $^{a}$ \\ ${ }^{a}$ Department of Computing Science, ${ }^{b}$ Department of Oncology, University of Alberta, Canada
}

\begin{abstract}
The estimation of body composition (i.e., proportions of muscle and fat tissues) in cancer patients has important clinical and research applications. In particular, chemotherapy drug dosage is determined after taking into account the muscle and fat proportions in the patient's body. Recently, there has been considerable interest in studying the correlation between survival and body composition in cancer patients. We propose a fully automated framework for segmentation and quantification of muscle and fat tissues in thoracic CT images. A novel approach based on statistical deformation model (SDM) constrained deformable registration using the finite element method (FEM) is proposed. We obtained very good segmentation results with Jaccard scores of $94.95 \%$ for muscle and $94.82 \%$ for fat tissues respectively on a large data set of 116 thoracic CT images.
\end{abstract}

Index Terms - FEM registration, CT images, Muscle segmentation

\section{INTRODUCTION}

The muscle and fat tissues are target locations for the water- and fat-soluble drugs respectively used for cancer treatment. Consequently, the proportions of these tissues are believed to determine the chemotherapy toxicity and efficacy. Therefore, the estimation of muscle and fat tissue proportions is an important task in research studies related to cancer prognosis and treatment. Currently, this task is being addressed through the manual segmentation of muscle and fat tissue regions in computer tomography (CT) images taken at specific skeletal landmarks (which are good correlates of the whole body muscle and fat mass), using the pre-defined windows of Hounsfield units (HU, units of radiation attenuation) for each tissue [1], [2]. However, the manual segmentation of large databases of CT images used in these studies is not practical and hence automatic segmentation methods are needed. Although, the segmentation of the fat region using automatic methods [3], [4] is relatively straightforward due to the unique HU range of the fat tissue $[-190,-30]$, the automatic segmentation of the muscle region is quite challenging as there exists significant overlap between the HU ranges of the muscle tissue $[-29,150]$ and surrounding organs (see Figure 1c).

In cancer research studies that require the estimation of muscle and fat proportions, two types of CT images are considered, the abdominal and the thoracic CT images taken at the 3rd lumbar vertebra (L3) and the 4th thoracic vertebra (T4) respectively. The automatic segmentation of abdominal CT images has already been addressed in [5] with great success, where the difficult step of segmenting the muscle region was achieved by taking advantage of the well defined muscle shape present in abdominal CT images using a shape prior based segmentation method. But, this approach cannot be used for the segmentation of muscle regions in thoracic CT images as in the thoracic CT images the shape of the muscle does not conform to a

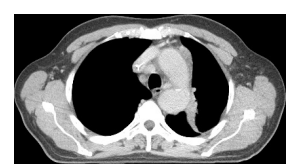

(a) Original $\mathrm{CT}$

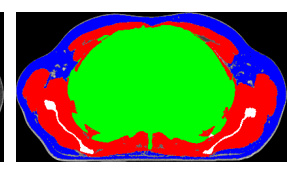

(b) Manual segm.

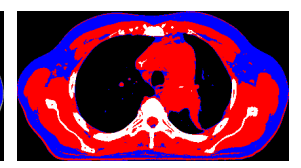

(c) Thresholded segm.
Fig. 1: Illustration of the challenge in thoracic CT segmentation. Muscle (red), Fat (blue), Inside region (green). It can be seen in (c) that segmentation solely based on thresholding the muscle and fat HU ranges results in a lot of errors due to the significant overlap of intensities between the muscle and the inside regions.

specific class of shapes (see Figure 4). However, in thoracic CT images the "inside" region containing the lungs, ribs and other organs (see Figure 1) exhibits a consistent shape across the patient population. Hence, a priori shape knowledge about the inside region's shape can be used to disambiguate the muscle tissue from the other organs in the inside region that have overlapping intensities. Based on this idea, we propose an automatic segmentation framework for thoracic CT images, where the inside region is first segmented using a shape prior model learned from training data and then the muscle and fat regions are obtained by thresholding the rest of the image using their respective $\mathrm{HU}$ ranges.

In this paper, we take a template-based segmentation approach where a binary template encoding an initial shape is deformed via non-rigid or deformable registration to match the region of interest in the input image. The desired segmentation boundary is then implicitly defined by the contour of the initial shape and the deformation field estimated between the template and the input image. Existing works on template-based segmentation either use a non-parametric representation of the deformation field [6] or parametrize the deformation field using B-spline basis functions [7]. However, a common drawback in these works is the use of a uniform discretization of the problem domain. This is inefficient because the deformation field is computed with the same accuracy everywhere, even though detailed deformations are only needed along the contour of the initial shape in the template. Therefore, we propose a finite element method (FEM) based registration framework to solve the templatebased segmentation problem. Our method employs a non-uniform mesh well adapted to the contour of the initial shape in the template and uses Lagrange basis functions instead of the B-spline basis functions to parametrize the deformation field. To better capture specific shape deformations, we extend the proposed FEM-based framework through the incorporation of a statistical deformation model (SDM) [8] learned from training data. There exist a few works on templatebased segmentation which follow a SDM constrained registration framework [9], [10], but these methods also suffer from the disadvantage of using a uniform mesh. 


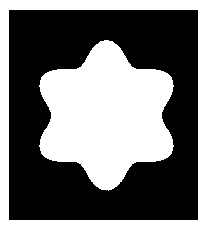

(a) Input image

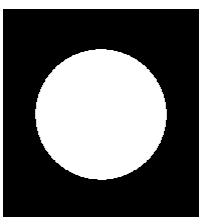

(b) Template

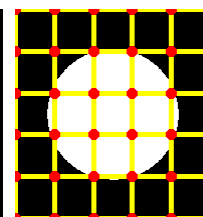

(c) Uniform mesh

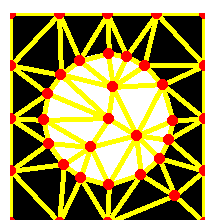

(d) Non-uniform mesh

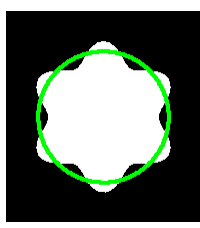

(e) Initial template contour

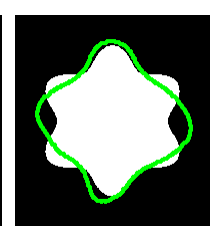

(f) Final contour (Bspline)

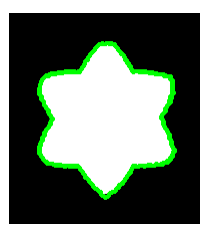

Final contou
Fig. 2: Comparison of FEM-based segmentation performance using a uniform (Bspline basis) VS non-uniform (Lagrange basis) mesh with the same number $(N=36)$ of nodes.

The main contributions in this work are given below:

(1) We propose an efficient FEM-based registration framework with a SDM using a non-uniform mesh (see Section 2) for inside region segmentation.

(2) A completely automatic segmentation framework for muscle and fat tissues in thoracic CT images (see Section 3) (such a framework has not been reported before to the best of our knowledge).

(3) We validated the proposed segmentation framework on a large number $(=116)$ of thoracic CT images and obtained excellent Jaccard scores: $94.95 \%$ for muscle and $94.82 \%$ for fat tissues respectively (see Section 4).

\section{SEGMENTATION VIA FEM-BASED DEFORMABLE REGISTRATION WITH A GAUSSIAN SDM}

\subsection{FEM-based deformable registration framework}

Given input $I: \Omega \rightarrow \mathbb{R}$ and template $I_{T}: \Omega_{T} \rightarrow \mathbb{R}$ images, where $\Omega, \Omega_{T} \subset \mathbb{R}^{d}$, the task of deformable or non-rigid registration is to find a dense deformation field $\mathbf{U}: \Omega_{T} \rightarrow \mathbb{R}^{d}$ such that the input image warped using the deformation field, $I(\mathbf{x}+\mathbf{U}(\mathbf{x}))$ is similar to the template image $I_{T}$. In a FEM-based framework, the deformation field $\mathbf{U}$ is approximated as a linear combination of a set of basis functions $\left\{\phi_{n}\right\}_{n=1}^{N}$ :

$$
\mathbf{U}(\mathbf{x})=\sum_{n=1}^{N} \mathbf{U}_{n} \phi_{n}(\mathbf{x}) \quad \forall \mathbf{x} \in \Omega_{T} .
$$

The basis functions $\left\{\phi_{n}\right\}_{n=1}^{N}$ are defined on a uniform or nonuniform tessellation of the template image domain $\Omega_{T}$ given by the mesh $\mathcal{M}=\left(\left\{P_{n}\right\}_{n=1}^{N}, \Delta\right)$, where $\left\{P_{n}\right\}_{n=1}^{N}$ denotes the nodes of the mesh and $\Delta$ is the set of elements (triangles or rectangles - see Figures 2c, 2d). The deformable registration task is transformed into finding the unknown nodal deformation field parameters $\Theta=\left[\mathbf{U}_{n}\right]_{n=1}^{N} \in \mathbb{R}^{N d}$ (which correspond to the value of the deformation field at the nodes $P_{n}$, i.e., $\mathbf{U}_{n} \equiv \mathbf{U}\left(P_{n}\right)$ ) through the finite-dimensional multivariate minimization of an energy:

$$
\Theta^{*}=\underset{\Theta \in \mathbb{R}^{N d}}{\operatorname{argmin}} E_{D}\left(\Theta ; I, I_{T}\right)+\alpha E_{R}(\Theta),
$$

where $E_{D}$ is the data term which measures the similarity between the warped input and the template images, $E_{R}$ is the regularization term that enforces the smoothing constraints on the estimated deformation field and $\alpha$ is the regularization constant. Choosing the data and regularization terms as the sum of squared differences (SSD) similarity measure and the diffusion regularizer $\frac{1}{2} \sum_{i=1}^{d} \int_{\Omega_{T}} \nabla U_{i}^{\mathrm{T}} D \nabla U_{i} d \mathbf{x}$ [11] respectively, we get the finitedimensional formulations of these terms using the FEM approximation in (1) as:

$$
\begin{gathered}
E_{D}^{\mathrm{SSD}}\left(\boldsymbol{\Theta} ; I, I_{T}\right)=\int_{\Omega_{T}}\left(I\left(\mathbf{x}+\sum_{n=1}^{N} \mathbf{U}_{n} \phi_{n}\right)-I_{T}(\mathbf{x})\right)^{2} d \mathbf{x}, \\
E_{R}^{\mathrm{diff}}(\mathbf{\Theta})=\sum_{i=1}^{d} \sum_{m, n=1}^{N} U_{n i} U_{m i} \int_{\Omega_{T}} D\left(\nabla \phi_{n} \cdot \nabla \phi_{m}\right) d \mathbf{x},
\end{gathered}
$$

where $\mathbf{U}_{n}=\left[U_{n i}\right]_{i=1}^{d}$ and $D$ is the diffusivity or the stiffness field. A common way of minimizing (2) is to decouple the data and regularization terms and minimize them alternatively [12]. However, here we perform a combined minimization of the data and regularization terms using a gradient-descent strategy $\Theta^{(k+1)}=\Theta^{(k)}-$ $\tau\left(\nabla E_{D}^{\mathrm{SSD}}\left(\Theta^{(k)} ; I, I_{T}\right)+\alpha \nabla E_{R}^{\text {diff }}\left(\Theta^{(k)}\right)\right)$, where $\nabla \equiv \nabla \Theta$ and $\tau$ the time step is determined using line search.

\subsection{Template-based segmentation via FEM-based registration}

In order to perform template-based segmentation using the above described FEM-based registration framework, a binary image $I_{T}$ : $\Omega_{T} \rightarrow\{0,1\}$ defining an initial shape of the region of interest is chosen as the template. This template is then deformed through the energy minimization in (2) and the final segmentation label $I^{*}$ : $\Omega \rightarrow\{0,1\}$ is given by inverse warping the template using the optimal nodal deformation parameters $\boldsymbol{\Theta}^{*}=\left\{\mathbf{U}_{n}^{*}\right\}_{n=1}^{N}$ as:

$$
I^{*}(\mathbf{x})=I_{T}\left(\mathbf{x}-\sum_{n=1}^{N} \mathbf{U}_{n}^{*} \phi_{n}\right) \quad \forall \mathbf{x} \in \Omega .
$$

Different types of basis functions can be used for the parameterization of the deformation field in (1). Here, we propose the use of piecewise-linear Lagrange basis functions (hat functions) [13] instead of the popular cubic B-spline basis functions [7]. This is because the Lagrange basis can be naturally defined on non-uniform meshes whereas the standard cubic B-spline basis is restricted to uniform meshes. As mentioned earlier, the use of a non-uniform mesh adapted to the contours of the initial shape in the template leads to greater computational efficiency over a uniform mesh. We illustrate this fact using a simple example in Figure 2, where the "star" shaped region of interest in a $\sim 200 \times 200$ input image is successfully segmented using a Lagrange basis on a non-uniform mesh generated from just 36 nodes (see Figures 2d, 2g) whereas the use of a Bspline basis on a uniform mesh with the same number of nodes (see Figures $2 c, 2 f$ ) results in a failed segmentation. Hence, for the same amount of computational effort, i.e., the number of nodal deformation parameters needed to be estimated, the use of Lagrange basis on a non-uniform mesh is much more accurate than the use of B-spline basis on a uniform mesh. 


\subsection{Gaussian statistical deformation model}

Let us assume that we obtained a set of $M$ nodal deformation field parameters $\left\{\boldsymbol{\Theta}^{(m)}=\left[\mathbf{U}_{n}^{(m)}\right]_{n=1}^{N}\right\}_{m=1}^{M}$ by registering $M$ training images $\left\{I^{(m)}: \Omega \rightarrow \mathbb{R}\right\}_{m=1}^{M}$ to a template image $I_{T}$, using the above discussed FEM-based deformable registration method. For introducing a priori shape knowledge into the future registration tasks, we now construct a statistical deformation model (SDM) from these nodal deformation field parameters $\left\{\Theta^{(m)}\right\}_{m=1}^{M}$. Following [14], the space of deformation parameters is modeled using a multivariate Gaussian density $\mathcal{N}\left(\overline{\boldsymbol{\Theta}}, \Sigma_{\boldsymbol{\Theta}}\right)$, with a sample mean $\overline{\boldsymbol{\Theta}}$ and a $N d \times N d$ sample covariance matrix $\Sigma_{\Theta}$. Further, the dominant modes of shape variation are computed using principal component analysis (PCA) and they are used to devise an additional shape-based regularization term as follows:

$$
\begin{aligned}
& E_{S}^{\mathrm{PCA}}(\boldsymbol{\Theta})=\|\mathbf{B}(\boldsymbol{\Theta}-\overline{\boldsymbol{\Theta}})\|^{2}+\frac{1}{2\left(\beta \sigma_{0}^{2}\right)}\|\boldsymbol{\Theta}-\overline{\boldsymbol{\Theta}}\|^{2}, \\
& \mathbf{B}=\operatorname{diag}\left(\eta_{1} \ldots \eta_{K}\right)\left[B_{1} \ldots B_{K}\right]^{\mathrm{T}}, \\
& \underline{\Sigma}_{\boldsymbol{\Theta}}=(1-\beta) \Sigma_{\boldsymbol{\Theta}}+\beta \sigma_{0}^{2} \operatorname{Id}_{N d}, \\
& \eta_{k}^{2}=\left((1-\beta) \sigma_{k}^{2}+\beta \sigma_{0}^{2}\right)^{-1}-\left(\beta \sigma_{0}^{2}\right)^{-1},
\end{aligned}
$$

where, $\left\{B_{k}\right\}_{k=1}^{K}$ is the PCA basis corresponding to the augmented covariance matrix $\underline{\Sigma}_{\Theta}$. Here, $\sigma_{k}^{2}$ are the eigen values of the matrix $\Sigma_{\Theta}$ and $\beta, \sigma_{0}$ are constants. The above regularizer can be seen as imposing a shape prior on the deformation fields by penalizing deviations from the Gaussian SDM as opposed to strictly restricting the deformation fields to the span of the PCA basis. Incorporating the shape-based regularizer into (2) we obtain the following statistically constrained FEM-based deformable registration formulation:

$$
\boldsymbol{\Theta}^{*}=\underset{\Theta \in \mathbb{R}^{N d}}{\operatorname{argmin}} E_{D}^{\mathrm{SSD}}\left(\boldsymbol{\Theta} ; I, I_{T}\right)+\alpha E_{R}^{\mathrm{diff}}(\boldsymbol{\Theta})+\beta E_{S}^{\mathrm{PCA}}(\boldsymbol{\Theta}),
$$

where $\alpha, \beta$ are regularization constants. Again, a line search based gradient descent strategy with an additional shape-based term $\nabla E_{S}^{\mathrm{PCA}}\left(\Theta^{(k+1)}\right)$ is used to perform the minimization of the above energy.

\section{PROPOSED FRAMEWORK FOR AUTOMATIC MUSCLE AND FAT TISSUE SEGMENTATION}

The main idea of the proposed segmentation framework is to first determine the "inside" region (see Figure 1b) of an input CT image through shape prior based segmentation using the FEM-based deformable registration with a Gaussian SDM presented in the Section 2.1. Then, the muscle and fat regions are segmented by thresholding the points in the input $\mathrm{CT}$ image not belonging to the inside region using the pre-defined HU ranges of muscle and fat tissues respectively. Before these segmentation steps are performed, a shape prior of the inside region shape is constructed from training data which involves the computation of a mean shape and encoding the deviations from the mean shape using a Gaussian SDM. The various components of the proposed segmentation framework are described in detail below:

(1) Computation of mean inside region shape: Given a training set of binary inside region shapes $\left\{L^{(m)}: \Omega \rightarrow\{0,1\}\right\}_{m=1}^{M}$, we follow the backward approach [15] for mean shape computation. In this approach, $L^{(1)}$ is chosen (without loss of generality) as the reference shape and all the other training shapes $\left\{L^{(m)}\right\}_{m=2}^{M}$ are non-rigidly registered (after an initial

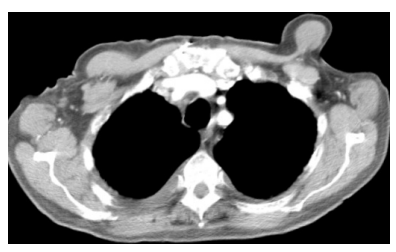

(a)

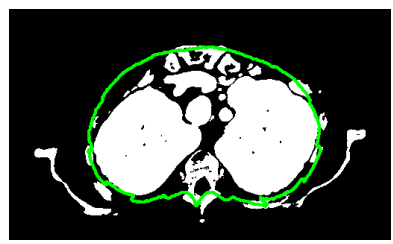

(c)

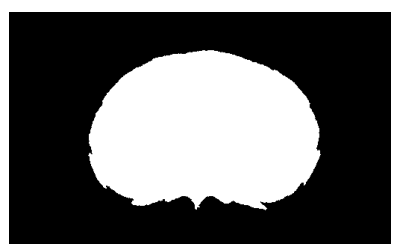

(b)

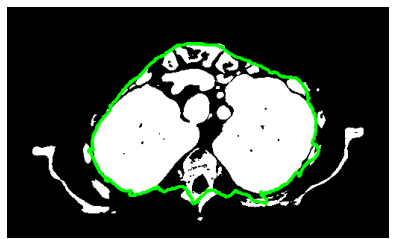

(d)
Fig. 3: (a) Input image $I$ (b) Mean shape of the inside region $\bar{L}$ (c) Thresholded input image $B I$ initialized with $\bar{L}$ (green contour) (d) $B I$ with final estimated inside region shape $L^{*}$ (green contour)

affine alignment) to $L^{(1)}$ using the FEM-based deformable registration method (see Section 2.1). The unbiased mean inside region shape $\bar{L}$ (see Figure $3 b$ ) is obtained by warping back the average of the registered training shapes using the inverse of the average deformation field.

(2) Building a SDM of inside region shape: The possible variations of the inside region shape are represented by the set of nodal deformation field parameters $\left\{\boldsymbol{\Theta}^{(m)}\right\}_{m=1}^{M}$ that are estimated through the FEM-based deformable registration (after an initial affine alignment) of the training shapes $\left\{L^{(m)}\right\}_{m=1}^{M}$ with the mean shape $\bar{L}$. A Gaussian SDM is built to compactly encode these shape variations and the corresponding PCA-based regularizer $E_{S}^{\mathrm{PCA}}$ (Equation (6)) is formulated for enforcing shape prior constraints in the next step.

(3) Segmentation via SDM constrained FEM-based registration: The segmentation of the inside region in the input image $I$ is performed on a binary thresholded version of the input image $B I: \Omega \rightarrow\{0,1\}$, which is obtained by setting the points in the input image that lie outside the HU ranges of muscle and fat tissues to 1 . In order to initialize the segmentation, the mean shape $\bar{L}$ is affinely-aligned with the thresholded image $B I$ (see Figure $3 \mathrm{c}$ ). The mean shape $\bar{L}$ is then deformed towards the thresholded image $B I$ according to the minimization of the energy in (7) incorporating the shape-based regularizer $E_{S}^{\mathrm{PCA}}$. The desired final inside region shape $L^{*}$ (see Figure 3d) is determined by warping back the mean inside region shape $\bar{L}$ using the inverse of the optimal nodal deformation field parameters (see Equation (5)).

(4) Muscle and fat region segmentation: The muscle and fat region segmentation is performed by thresholding points in the input image that do not belong to the estimated inside region using the respective muscle and fat HU ranges (see Figure 4).

\section{EXPERIMENTS}

Our experimental data set consisted of 146 axial 2D thoracic CT images of size $512 \times 512$ taken at the level of T4 from patients with head and neck cancers. Out of these 30 images were used for train- 


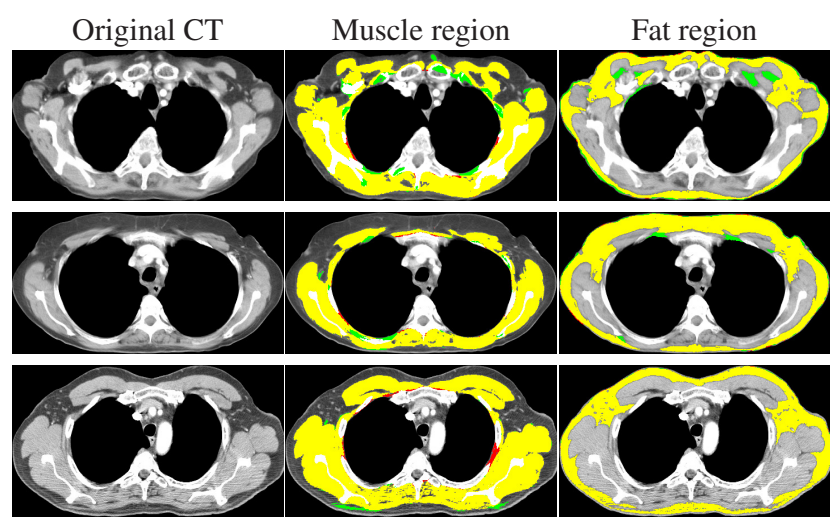

Fig. 4: Muscle and fat region segmentation results. Manual label (red), automatic label (green) and overlap (yellow).

\begin{tabular}{|c|c|c|}
\hline \multirow{2}{*}{ Tissue } & \multicolumn{2}{|c|}{ Jaccard (\%) } \\
\cline { 2 - 3 } & Thresholding & Proposed framework \\
\hline Muscle & $86.82 \pm 5.31$ & $\mathbf{9 4 . 9 5} \pm \mathbf{2 . 1 0}$ \\
\hline Fat & $87.51 \pm 8.74$ & $\mathbf{9 4 . 8 2} \pm \mathbf{5 . 0 5}$ \\
\hline
\end{tabular}

Table 1: Comparison of Jaccard scores obtained by thresholdingbased segmentation and the proposed FEM-based segmentation framework with a SDM on 116 CT images. (All values are reported as mean $\pm \mathrm{SD}$ )

ing the SDM. We evaluated the performance of the proposed automatic segmentation framework by comparison with manual segmentations on the remaining 116 images in our data set. The manual segmentations were performed by a single expert operator using SliceO-Matic V4.3 software (Tomovision, Montreal, Canada), while the proposed automatic framework was implemented in MATLAB. The regularization parameters were chosen as $\alpha=1.5$ and $\beta=1.0$ (see Equation 7). The non-uniform mesh on the mean shape of the inside region consisted of 503 nodes. It was generated by manually selecting a few seed point along the contour of the mean shape followed by Delaunay triangulation and refinement. We ran the experiments on a $3.2 \mathrm{GHz}$ Quadcore machine with $4 \mathrm{~GB}$ of RAM. The automatic segmentation took about $1 \mathrm{~min}$ for each image. We used the Jaccard score metric to measure the overlap between the manual and automatic segmentations. In Table 1, it can be seen that a significant improvement in the Jaccard scores is achieved using our proposed framework compared to the simple thresholding-based segmentation. In Figure 4, visual segmentation results obtained using our proposed framework are shown on 3 images. Further, we also estimated the tissue cross-sectional areas $\left(\mathrm{cm}^{2}\right)$ from segmentations by summing up tissue pixels and multiplying by the pixel surface area. In Table 2, we can see that the coefficient of variation $(\mathrm{COV})$ between the automatic and manual methods is within $(1-3) \%$ which is similar or less than inter- or intra- operator COVs reported for manual segmentation [16].

\section{CONCLUSION}

The proposed automatic framework provides fast and accurate segmentations of both the muscle and fat tissues, which permits the undertaking of large scale cancer research studies involving measures based on muscle and fat proportions in the human body.

\begin{tabular}{|c|c|c|c|}
\hline Tissue & \multicolumn{2}{|c|}{ Area $\left(\mathrm{cm}^{2}\right)$} & COV \\
\cline { 2 - 3 } & Manual & Automatic & $(\%)$ \\
\hline Muscle & $197.8 \pm 44.7$ & $199 \pm 45.2$ & $1.39 \pm 1.58$ \\
\hline Fat & $168.1 \pm 84.4$ & $172.5 \pm 90$ & $2.57 \pm 3.03$ \\
\hline
\end{tabular}

Table 2: Comparison of tissue cross-sectional areas estimated using manual and automatic segmentations on $116 \mathrm{CT}$ images. (All values are reported as mean $\pm \mathrm{SD}$ )

\section{REFERENCES}

[1] C.M.M. Prado, V.E. Baracos, L.J. McCargar, M. Mourtzakis, K.E. Mulder, T. Reiman, and et al., "Body composition as an independent determinant of 5-fluorouracil-based chemotherapy toxicity," Clin. Canc. Res., vol. 13, no. 11, pp. 3264-3268, 2007.

[2] C.M.M. Prado, J.R. Lieffers, L.J. McCargar, T. Reiman, M.B. Sawyer, and et al., "Prevalence and clinical implications of sarcopenic obesity in patients with solid tumours of the respiratory and gastrointestinal tracts: a population-based study," Lancet Oncol., vol. 9, no. 7, pp. 629-635, 2008.

[3] A.N. Bandekar, M. Naghavi, and I.A. Kakadiaris, "Performance evaluation of abdominal fat burden quantification in ct," in IEEE-EMBS 2005, 2006, pp. 3280-3283.

[4] B. Zhao, J. Colville, J. Kalaigian, et al., "Automated quantification of body fat distribution on volumetric computed tomography," Jour. of comp. assis. tomography, vol. 30, no. 5, pp. $777,2006$.

[5] H. Chung, D. Cobzas, J. Lieffers, L. Birdsell, and V. Baracos, "Automated segmentation of muscle and adipose tissue on ct images for human body composition analysis," in SPIE Medical Imaging, 2009.

[6] Kinda Anna Saddi, Christophe Chefd'hotel, Mikael Rousson, and Farida Cheriet, "Region-based segmentation via non-rigid template matching," in MMBIA, 2007.

[7] C. Li and Y. Sun, "Active image: A shape and topology preserving segmentation method using b-spline free form deformations," in ICIP. IEEE, 2010, pp. 2221-2224.

[8] D. Rueckert, A.F. Frangi, and J.A. Schnabel, "Automatic construction of 3D statistical deformation models using non-rigid registration," in MICCAI, 2001, pp. 77-84.

[9] X. Huang, Z. Li, and D. Metaxas, "Learning coupled prior shape and appearance models for segmentation," MICCAI, pp. 60-69, 2004

[10] M. Taron, N. Paragios, and M.P. Jolly, "Registration with uncertainties and statistical modeling of shapes with variable metric kernels," TPAMI, vol. 31, no. 1, pp. 99-113, 2009.

[11] J. Modersitzki, Numerical methods for image registration, Oxford Univ. Press, 2004

[12] K. Popuri, D. Cobzas, and M. Jagersand, "Fast fem-based nonrigid registration," in $C R V, 2010$, pp. 378-385.

[13] O.C. Zienkiewicz and R.L. Taylor, The finite element method for solid and structural mechanics, Elsevier ButterworthHeinemann, 2006.

[14] T. Albrecht, M. Luthi, and T. Vetter, "A statistical deformation prior for non-rigid image and shape registration," in CVPR, 2008, pp. 1-8.

[15] A. Guimond, J. Meunier, and J.P. Thirion, "Average brain models: A convergence study," CVIU, vol. 77, no. 2, pp. 192-210, 2000.

[16] E.W. Demerath, K.J. Ritter, W.A. Couch, N.L. Rogers, G.M. Moreno, et al., "Validity of a new automated software program for visceral adipose tissue estimation," Int. jour. of obesity, vol. 31, no. 2, pp. 285-291, 2006. 